\title{
Producción Fúngica de Proteasas Inducidas con Pelo de Cerdo
}

\author{
Imir R. Vázquez ${ }^{1}$, Antonio F. Aguilera ${ }^{1}$, Lilia A. Prado-Barragán² y Cristóbal N. Aguilar ${ }^{3}$ \\ (1) Universidad Autónoma Agraria Antonio Narro, Departamento de Nutrición y Alimentos, \\ Edificio UAAAN, Carretera Km 6, Buenavista, Saltillo, Coahuila-México \\ (e-mail: mvazque@sigma-alimentos.com) \\ (2) Universidad Autónoma Metropolitana-Iztapalapa, Departamento de Biotecnología, División \\ de Ciencias Biológicas y de la Salud. Av. Michoacán y La Purísima s/n, Col. Vicentina, \\ 09340 México, D.F.-México (e-mail: lapb@xanum.uam.mx) \\ (3) Universidad Autónoma de Coahuila, Facultad de Ciencias Químicas, Departamento de \\ Investigación en Alimentos, Blvd. V. Carranza y José Cárdenas, Col. República, Saltillo, \\ Coahuila-México (e-mail: cag13761@mail.uadec.mx)
}

\begin{abstract}
Resumen
Se evaluaron las condiciones de producción de proteasas fúngicas inducidas con pelo de cerdo usando dos sistemas de cultivo, el proceso de cultivo en estado sólido y el cultivo líquido sumergido. El medio de cultivo fue Czapek-Dox en ambos casos. La cinética fue monitoreada cada 24 horas durante un periodo de incubación de 120 horas. La actividad proteolítica fue evaluada por hidrólisis de azocaseína y la producción de la biomasa fue estimada por el método gravimétrico. Rhizopus oryzae fue el microorganismo capaz de utilizar el pelo de cerdo como única fuente de carbononitrógeno, produciendo en cultivo sumergido los mayores títulos de actividad queratinasa. Se determinó el efecto que tiene el $\mathrm{pH}$, el pretratamiento del sustrato y la adición de diferentes niveles de material queratinoso sobre el crecimiento fúngico y la actividad enzimática. Los resultados permitieron obtener títulos de actividad proteolítica de $975 \mathrm{U} / \mathrm{L}$.
\end{abstract}

Palabras clave: proteasas, Rhizopus oryzae, pelo de cerdo, producción fúngica

\section{Fungal Production of Proteases Induced with Pig Hair}

\begin{abstract}
The production of new proteases induced with pig hair was evaluated using two systems, submerged culture and solid state culture. Culture medium was Czapek-Dox for both systems. Enzyme production kinetic was monitored each 24 hours during 120 hours. Protelytic activity was evaluated with the azocaseín method and biomass production was determined by the gravimetric method. Rhizopus oryzae was the microorganism with the capacity of using the pig hair as the single carbonnitrogen source, producing in submerged fermentation the highest enzymatic titers. The effect of $\mathrm{pH}$, the substrate pretreatment and addition of different levels of substrate on proteolyitic activity and biomass production were determined. Results permitted to obtain titers of proteolytic activity of 975 $U / L$.
\end{abstract}

Keywords: protease, pig hair, Rhizopus oryzae, fungal production 


\section{INTRODUCCIÓN}

La generación de desechos agroindustriales representa una fuente de contaminación de alto impacto ecológico. De manera particular, la industria pecuaria asociada a rastros de ganado porcino, representa la producción de residuos de biodegradación lenta; por lo tanto es necesario tratarlos para disminuir su potencial contaminante (Bertsch y Coello, 2005). Los bioprocesos representan una atractiva alternativa considerada como una tecnología limpia, rentable y de fácil manejo, en donde es posible utilizar estos desechos como fuentes inductoras para el desarrollo de proteasas fúngicas. En este sentido, un gran avance en la biodegradación de plumas de gallina y desechos de cordero ha sido alcanzado (Brandelli y Rifle, 2005; Grazziotin et al., 2007)

El pelo de cerdo representa una fuente de proteínas muy importante lo que permite que pueda ser utilizado como sustrato en fermentaciones para la obtención de proteasas y su posible aplicación en industrias alimenticias, agroquímicas o bebidas. La biodegradación del material queratinoso por microorganismos que poseen actividad queratinolítica representa un método alternativo para aumentar el valor nutricional de los desechos queratinosos. La proteína estructural (queratina) puede ser degradada por la enzima queratinasa, producida por especies de hongos parásitos saprofitos, algunas cepas de Bacillus y algunos actinomicetos (Pokorny et al. 1979; Vitale et al. 1986; Renko et al. 1981, 1989; Bascaran et al. 1990; Yeoman y Edwards,1994).

Las proteasas constituyen uno de los grupos más importantes de las enzimas industriales. En los años recientes, el uso de la proteasa alcalina en una variante del proceso industrial involucra detergentes, alimentos, tratamiento de pieles y seda (Kembhavi et al., 1993). Actualmente una gran proporción disponible de las proteasas comerciales son derivadas de cepas de Bacillus; aunque también las de origen fúngico han sido empleadas y representan 30 del $40 \%$ del costo de producción de las enzimas industriales. Las proteasas son enzimas de importancia central debido a que ocupan una posición primaria en ambos campos; tanto el fisiológico como el comercial ya que son ampliamente usadas en alimentos; (bebidas, panificaciones, ablandamiento de carne, síntesis del aspartame, detergente e industrias peleteras). Adicionalmente, una variedad de proteasas contienen importantes aplicaciones farmacéuticas. Las enzimas proteoliticas pueden ser usadas después de un pretratamiento químico o de cocimiento con vapor de los desechos ricos en queratina. Este proceso requiere cantidades significantes de energía y algunas veces se destruyen aminoácidos valiosos tales como la valina y el triptòfano (Chandrasekaran y Dhar 1986). Los enlaces disulfuro en la queratina inciden en su degradación, además, la desnaturalización del sustrato insoluble y el rompimiento de los enlaces del disulfuro parece ser necesario para hacer accesible la queratina a la enzima hidrolítica excretada. Por lo tanto se requiere del desarrollo de procesos de tratamiento que no afecten la calidad de los productos de la hidrólisis enzimática.

Ignatova et al. (1999), reportaron la producción y caracterización de una queratinasa termofílica demostrando que las propiedades de las enzimas degradadoras de queratina difieren entre especies. En el estudio se empleo una cepa termofílica del actinomiceto, Thermoactinomycetes candidus, aislada de su medio (lodos de alcantarillado). Los microorganismos termofílicos representan una fuente muy atractiva para la producción de actividades proteolíticas tal como lo sugieren diversos reportes. Las proteasas obtenidas de microorganismos termofílicos muestran temperaturas óptimas para su actividad en el rango de $70-100{ }^{\circ} \mathrm{C}$ y un alto grado de termo-estabilidad. Las enzimas de microorganismos termófilos, han despertado una creciente atención entre los investigadores por 2 razones fundamentales: primero, sus propiedades únicas $y$, especialmente la alta estabilidad estructural, es lo que han hecho que estas enzimas sean consideradas como extremadamente importantes en muchos procesos industriales. Segundo, éstas son de interés considerable para procesos donde su característica de termo-estabilidad sea necesaria (Farag y Hassan 2003; Malathi y Chakraborty 1991; Muro et al. 1991; Ozawa et al. 1996).

La queratina es el mayor componente del pelo, plumas y lana. Las industrias porcícolas no poseen la infraestructura necesaria para tratar toda la gama de residuos que genera, representando un foco severo de contaminación y riesgo sanitario. Con el fin de generar alternativas de tratamiento del pelo de cerdo, el objetivo del presente trabajo fue evaluar las condiciones de producción de la enzima queratinasa fúngica en dos sistemas de cultivo, analizando el efecto que tiene el $\mathrm{pH}$, el 
pretratamiento del sustrato y la adición de diferentes niveles de material queratinoso sobre el crecimiento fúngico y la actividad enzimática.

\section{METODOLOGÍA.}

\section{Materia prima}

El material queratinoso (pelo de cerdo) fue proporcionado por el rastro municipal de la ciudad de Saltillo, Coahuila, México. Éste, fue lavado para limpiar restos de contaminantes, posteriormente fue sometido a una esterilización en auto clave a $\left(121^{\circ} \mathrm{C}\right.$ por $15 \mathrm{~min}$.) para eliminar los microorganismos presentes. El material queratinoso esterilizado fue sometido a una reducción de tamaño de partícula, para cortar el pelo en un rango de 0.5 a $1.0 \mathrm{~cm}$ de longitud, posterior mente se utilizó un molino de martillo para reducir aun más el tamaño de partícula hasta quedar en una medida de 0.1 y $0.2 \mathrm{~cm}$ de longitud. Para aquellos experimentos en que se requirió desgrasar el material queratinoso se utilizaron dos solventes, hexano y éter de petróleo.

\section{Caracterización de la materia prima}

El material queratinoso se caracterizó aplicando los procedimientos descritos en el AOAC (1980), para un análisis bromatológico que incluyó los parámetros físico-químicos siguientes: materia seca total, contenido de humedad, cenizas, proteínas, grasa, fibra cruda, azucares totales y reductores.

\section{Selección del microorganismo}

Trece microorganismos fueron evaluados por su capacidad de producción de proteasas. Se emplearon cepas de Aspergillus, Penicillium, y Rhizopus, pertenecientes a colecciones de la UAMIIRD y de la UAdeC (Cruz-Hernández et al. 2005). Rhizopus oryzae fue el microorganismo que demostró una mayor capacidad de adaptación al medio cuya única fuente de carbono y energía era el pelo de cerdo en cultivos sumergido. Adicionalmente este hongo se sometió a un análisis de la capacidad de invasión y determinación de su velocidad de crecimiento radial.

El experimento se llevó a cabo en placas de Petri de $8 \mathrm{~cm}$ de diámetro, conteniendo medio Czapekdox suplementado con pelo de cerdo y agar, previamente autoclavado; se inoculó el centro de la placa con las esporas del hongo y se incubo a $30^{\circ} \mathrm{C}$, haciendo evaluaciones cada $12 \mathrm{~h}$. del crecimiento radial por los días que fuera necesario.

\section{Propagación de inóculos}

Las esporas de $R$. oryzae se inocularon sobre $30 \mathrm{ml}$ de agar PDA contenidos en matraces Erlenmeyer de $250 \mathrm{ml}$ y estos se incubaron a $30^{\circ} \mathrm{C}$ por 5 días. Las esporas producidas se cosecharon con una solución de Tween 80 al $0.01 \%$.

\section{Medios de cultivo}

Para evaluar la producción de la enzima queratinasa fúngica se empleo el medio mínimo Czapek Dox tanto en cultivo en medio sólido (CMS) como en cultivo líquido sumergido (CML). La composición del medio (\%) fue: $\mathrm{NaNO}_{3}(0.25), \mathrm{KH}_{2} \mathrm{PO}_{4}(0.1), \mathrm{MgSO}_{4}-7 \mathrm{H}_{2} \mathrm{O}(0.05), \mathrm{KCl}(0.05)$ y pH de 6.0. La fuente de carbono fue el pelo de cerdo añadido como inductor de la actividad queratinasa. Se utilizaron concentraciones de pelo de cerdo de $0.25,0.50,0.70,10$ y $20 \%$ para el CML y de $70 \%$ para el cultivo en medio sólido (CMS). El material se evaluó con y sin grasa como soporte e inductor para la producción de la enzima queratinasa. La adición de un pulso de solución de glucosa a 1, 5 y $10 \mathrm{~g} / \mathrm{L}$ fue considerada como factor de activación de crecimiento.

Cultivo en medio sólido (CMS) y cultivo líquido sumergido (CML).

Se utilizaron placas de Petri de $8 \mathrm{~cm}$. que contenían la cantidad establecida de pelo de cerdo el cual fue impregnado con $10 \mathrm{ml}$ de medio Czapek-Dox ajustados a un $\mathrm{pH}$ de 6.0. Enseguida estas fueron 
esterilizadas. Bajo condiciones estériles, se inoculados con $20 \mu$ de esporas de Rhizopus oryzae, ajustadas a una concentración de $5 \times 10^{7}$ esporas por gramo de soporte para las respectivas concentraciones. Finalmente se incubaron a una temperatura de $30^{\circ} \mathrm{C}$ por $120 \mathrm{~h}$. Se utilizaron matraces Erlenmeyer de $250 \mathrm{~mL}$ con la cantidad determinada de pelo de cerdo y $50 \mathrm{~mL}$ de medio de cultivo líquido Czapek Dox ajustado a pH de 6.0. En seguida se esterilizaron en un autoclave. Bajo condiciones estériles se inocularon con $20 \mu \mathrm{l}$ de esporas de $R$. oryzae, ajustadas a una concentración de esporas $5 \times 10^{7}$ por $\mathrm{ml}$ de medio Czapek Dox. Los matraces fueron incubados a una temperatura de $30^{\circ} \mathrm{C}$ por un periodo de $120 \mathrm{~h}$. en agitación constante de $220 \mathrm{rpm}$. La medición de la biomasa se llevo a cabo por el método gravimétrico (AOAC, 1980). La actividad proteolítica queratinasa fue ensayada por la técnica de la azocaseina. Brevemente, el método consiste en colocar $10 \mathrm{mg}$ de azocaseina en tubos de ensaye, luego se agrega $1 \mathrm{ml}$ de solución buffer fosfato de $\mathrm{pH} 7$ y $3 \mathrm{ml}$ de agua destilada, enseguida se incuban a $37^{\circ} \mathrm{C}$ en baño maría, con agitación constante. Después de los 30 minutos de incubación, se filtra en papel Whatman 41, esto con el fin de detener la reacción, finalmente el líquido filtrado se colecta en tubos de ensayo y se lee en el espectrofotómetro a 520 $\mathrm{nm}$. Para el control, se realizan los mismos procedimientos, pero sin añadir extracto enzimático. Una unidad enzimática queratinasa es definida como la cantidad de enzima que produce una absorbancia de 0.1 bajo las condiciones descritas anteriormente.

\section{RESULTADOS Y DISCUSIÓN}

En el presente estudio se evaluaron las condiciones preliminares de producción de la enzima queratinasa de $R$. oryzae sobre pelo de cerdo. Los resultados obtenidos se presentan en tres etapas, en la primera se incluyen los resultados obtenidos de la caracterización fisicoquímica y de caracterización del material queratinoso, en la segunda etapa se reportan los resultados de la selección de los microorganismos y de la caracterización de $R$. oryzae y en ultima etapa se reportan los resultados de las condiciones de producción de la queratinasa fúngica en CMS y CML.

\section{Primera etapa: caracterización fisicoquímica del pelo de cerdo}

Los resultados obtenidos de la caracterización fisicoquímica del pelo de cerdo se presenta en la Tabla 1; donde se puede observar claramente que el material posee un alto porcentaje de proteína (88\%), sin embargo, no posee carbohidratos o azucares simples de fácil asimilación por los microorganismos lo que probablemente genere la necesidad de complementar el medio de cultivo cuando el pelo de cerdo es utilizado como inductor de la actividad queratinasa. Los resultados del contenido proteico obtenidos en la presente investigación son mayores que los reportados en la literatura (Mahan y Shields, 1998), en donde se reportaron niveles de 55 a $70 \%$ de proteína contenida en el pelo del cerdo. Adicionalmente es importante añadir que los resultados obtenidos para el resto de los componentes concuerdan con la literatura citada, ya que los niveles de minerales están alrededor de $1 \%$ y la grasa entre 2 al $7 \%$. Además, este el primer reporte de los niveles de fibra cruda en el material, el cual es de alrededor de $0.4 \%$ y de humedad cercana al $3.5 \%$.

\section{Segunda etapa: selección del microorganismo y caracterización de la cepa seleccionada.}

La selección del microorganismo incluyó el crecimiento de cepas fúngicas sobre medios de cultivo en estado sólido y liquido con medio Czapek-Dox cuya fuente de carbono y nitrógeno, fue el pelo de cerdo. Las condiciones de cultivo incluyeron una temperatura de $30^{\circ} \mathrm{C}$, agitación en el caso de los cultivos líquidos y un monitoreo constante del crecimiento micelar. 10 de las 13 cepas evaluadas pertenecientes a la colección DIA/UAdeC (Cruz-Hernández et al, 2005) fueron incapaces de utilizar al material queratinoso en prueba como sustrato o fuente de carbono y nitrógeno, a excepción de los microorganismos: Aspergillus niger PSH, Penicillium commune ESS y Rhizopus oryzae (Tabla 2). Sin embargo este último fue capaz de crecer en los diferentes sistemas de cultivo empleados, tanto en estado sólido como en líquido de una manera diferentemente significativa sobre los otros dos microorganismos, por lo que se decidió utilizar la cepa de $R$. oryzae como fuente de la enzima queratinasa en este estudio. $R$. oryzae se caracterizó por mostrar un crecimiento rápido e invasivo sobre el material queratinoso, dependiente fuertemente de la disponibilidad de oxigeno ya que solo sobre superficies donde el material se encontraba en contacto directo con el aire produjo un micelio aéreo característico. 
Tabla 1: Caracterización físico-química del pelo de cerdo

\begin{tabular}{lc}
\hline Componente & $\%$ \\
\hline Humedad & 3.2 \\
Materia seca total & 96.8 \\
Cenizas & 0.73 \\
Proteína Cruda & 87.8 \\
Grasa & $7.3 \%$ \\
Fibra Cruda & 0.4 \\
Azucares totales & 0.5 \\
\hline
\end{tabular}

Tercera etapa: condiciones de producción de la queratinasa en cultivo líquido sumergido y sólido

La Figura 1 presenta los resultados de $\mathrm{pH}$ durante la producción de la enzima queratinasa en cultivo líquido sumergido. No hubo una diferencia significativa en el valor de $\mathrm{pH}$ cuando se empleo como sustrato pelo de cerdo con y sin pretratamiento para eliminar la capa protectora de grasa, además, el tipo de sistema de cultivo empleado no influye de manera significativa en los valores de $\mathrm{pH}$ (Figura 1a). Sin embargo, cuando se adicionaron pulsos de glucosa al CML, los valores de $\mathrm{pH}$ se incrementaron significativamente (Figura 1b), siendo este efecto mayor con un pulso de 5 que a 10 $\mathrm{g} / \mathrm{L}$. En CMS el pH no presentó variaciones significativas, la adición de glucosa a las muestras con y sin grasa no tuvo un efecto muy significativo en el comportamiento del pH durante la cinética de producción de la enzima queratinasa a diferencia del CML, ya que en las muestras sin el azúcar los valores de $\mathrm{pH}$ alcanzaron niveles de hasta 9 en las primeras $45 \mathrm{~h}$ de cultivo, incrementándose hasta alcanzar valores de $\mathrm{pH}$ de 11 al final de cultivo. Bajo estas condiciones, el crecimiento fúngico fue menor y más lento que en aquellas muestras complementadas con glucosa. Aparentemente el microorganismo produce carboxipeptidasas que generan una gran cantidad de grupos aminos que tienden a incrementar los valores de $\mathrm{pH}$. Además, la adición de un carbohidrato de fácil asimilación como lo es la glucosa modifica la fisiología del microorganismo, ya que a medida que se incrementa este azúcar en el medio los valore de $\mathrm{pH}$ tienden a ser menores, lógicamente es por la no producción de las carboxipeptidasas. Adicionalmente, es importante señalar que las muestras con grasa tendieron a alcalinizar más el medio de cultivo que las muestras sin grasas, y la utilización de la grasa a través de lipasas también puede tener un efecto serio y significativo sobre el comportamiento del $\mathrm{pH}$ del cultivo.

Los valores obtenidos en la presente investigación concuerdan con los reportados por Santos et al (1996), quienes obtuvieron los mismos patrones de incremento en los valores de $\mathrm{pH}$ cuando hicieron crecer a la cepa de Aspergillus fumigatus en medios cuya unica fuente de carbono y energia eran plumas de gallina. Se han reportado similares resultados obtenidos con cepas fungicas degradadotas de substratos keratinosos tales como las plumas de pollo y la lama de cordero (Mushin y Hadi, 2001). Este mismo comportamiento se obtuvo recientemente en los estudios reportados por Suntornsuk y Suntornsuk (2003), cuando evaluaron la degradación de las plumas de pollo por la cepa Bacillu sp Fk 46 , ya que obtuvieron valores de hasta 9 de $\mathrm{pH}$ cuando el crecimiento alcanzó sus maximos niveles. En el presente estudio, el efecto de la concentración inicial de pelo de puerco en el medio de cultivo fue evaluado en CML. La Figura 2 presenta los resultados obtenidos, los cuales indican que a mayor contenido de sustrato, el valor de la actividad enzimática es mas grande siendo un nivel de $30 \%$ de sustrato presente en el medio la concentración a la cual se alcanzan los mejores resultados. Es importante notar que no hubo efecto significativo del pre-tratamiento del pelo para remover la grasa.

La adición de glucosa promueve un retardo significativo en la producción de la enzima, siendo este efecto mayor a niveles superiores de glucosa en el medio. Los mejores resultados se obtienen con muestras sin tratar y con la adición de un pulso de glucosa de $5 \mathrm{~g} / \mathrm{L}$, ya que a las 120 horas de cultivo se alcanzan niveles de hasta $975 \mathrm{U} / \mathrm{mL}$, Aunque con un pulso de $1 \mathrm{~g} / \mathrm{L}$ de glucosa la actividad queratinasa es de $815 \mathrm{U} / \mathrm{L}$ a las $120 \mathrm{~h}$ de fermentación. 
Tabla 2: Capacidad de microorganismos para crecer en cultivos de pelo de cerdo

\begin{tabular}{lcc}
\hline Microorganismo & \multicolumn{2}{c}{ Crecimiento } \\
\cline { 2 - 3 } & Cultivo líquido & Cultivo sólido \\
\hline Penicillium commune GH2 & - & - \\
Penicillium commune EH2 & - & - \\
Penicillium commune EH3 & - & - \\
Penicillium commune ESS & + & + \\
Aspergillus terrícola PSS & - & - \\
Aspergillus ornatus ESH & - & - \\
Aspergillus rugulosa NS4 & - & - \\
Aspergillus fumigatus GS & - & - \\
Aspergillus rugulosa NH4 & - & - \\
Aspergillus niger PSH & ++ & ++ \\
Aspergillus niger GH1 & - & - \\
Aspergillus niger Aa-20 & - & - \\
Rhizopus oryzae & +++ & +++ \\
\hline
\end{tabular}

La Figura 3 presenta los resultados obtenidos de la determinación de la actividad enzimática queratinasa fúngica bajo diferentes concentraciones de glucosa, usando un valor de $30 \%$ de pelo sin pretratamiento para remoción de la grasa.

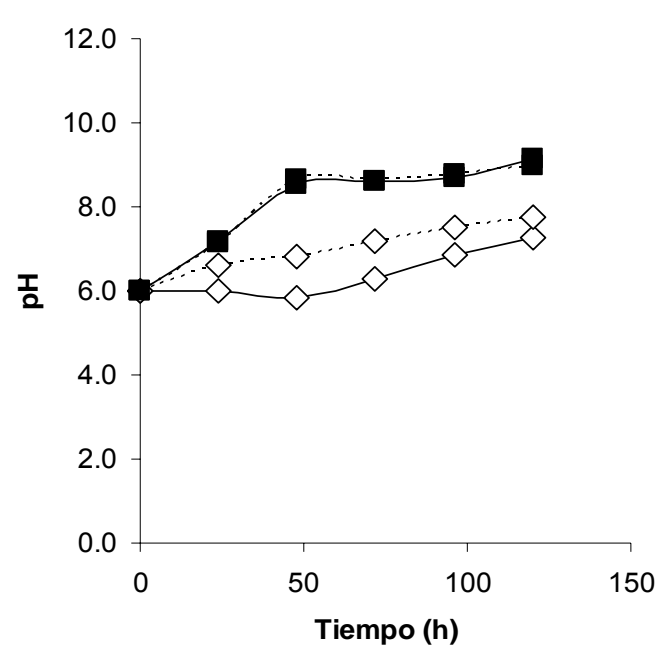

a

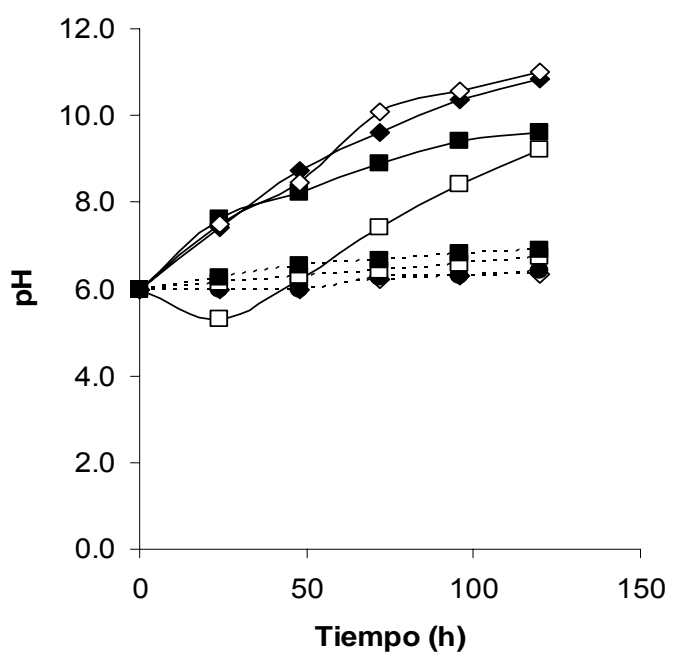

b

Fig. 1: Valores de pH durante la producción de queratianasa en CML (línea sólida) y CMS (línea

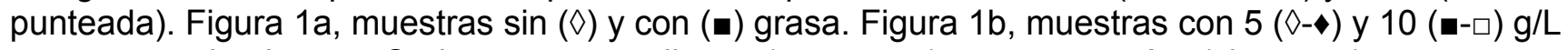
de glucosa. Serie con puntos llenos (con grasa) y puntos vacíos (sin grasa).

Estos resultados son mayores que los obtenidos por Santos et al. (1996), para A. fumigatus quien produjo la actividad hasta valores de $250 \mathrm{U} / \mathrm{L}$ y concuerdan con los reportados por Suntornsuk y Suntornsuk y Suntornsuk (2003), para Baciilus sp FK 46 quien produjo niveles de hasta 1000 U/L. y con lo reportado por Joo et al (2002), para Baciilus horikoschii quien produjo niveles de $800 \mathrm{U} / \mathrm{L}$. Los resultados obtenidos son menores que los niveles reportados por Mushin y Hadi (2001) para Trichophyton metagrophytes (8000 U/L), Aspergillis flavus (300 U/L), Microsporum gryseum (14000 u/L) y Chrysosporium pannicola (16000 U/L). En el presente estudio, la actividad proteolitica se presentó en ambos sistemas de cultivo, el CML como en CMS, además dicha actividad se detectó tanto con la presencia como con la ausencia de grasa en el pelo y con y sin la adición de glucosa, de tal manera que los resultados obtenidos nos permiten establecer que no es necesario pretratar al pelo de cerdo cuando este se emplee como fuente de carbono e inductor de la actividad deseada. A bajos niveles de concentración, la glucosa funcionó como un activador de la biomasa pero no como un iniciador de la capacidad queratinolitica del hongo. 


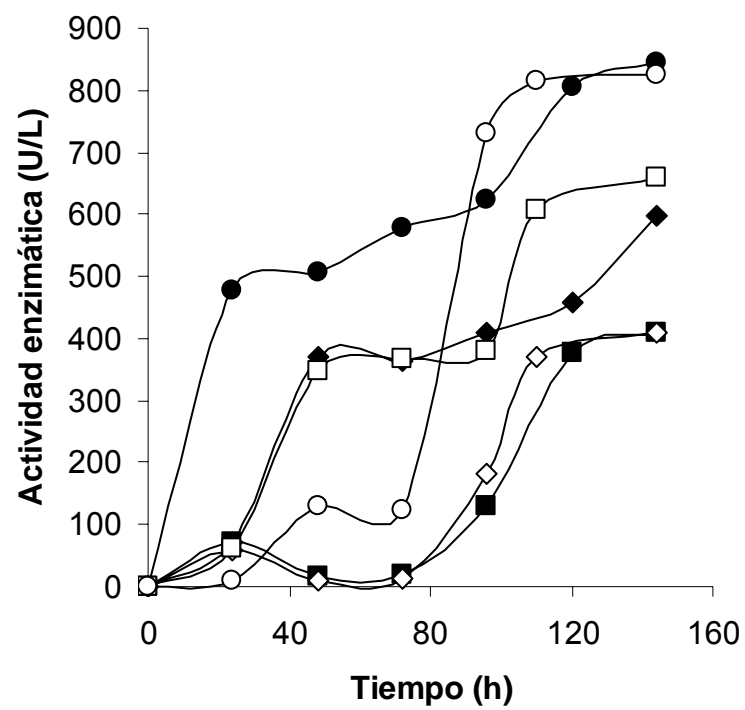

Fig. 2: Valores de actividad queratinasa fúngica a diferentes niveles de sustrato (pelo de cerdo) en CML. Los niveles de sustrato fueron: $10(\diamond), 20$

(匹)y $30(\bullet) \%$. Serie con puntos llenos (con grasa) y puntos vacíos (sin grasa)

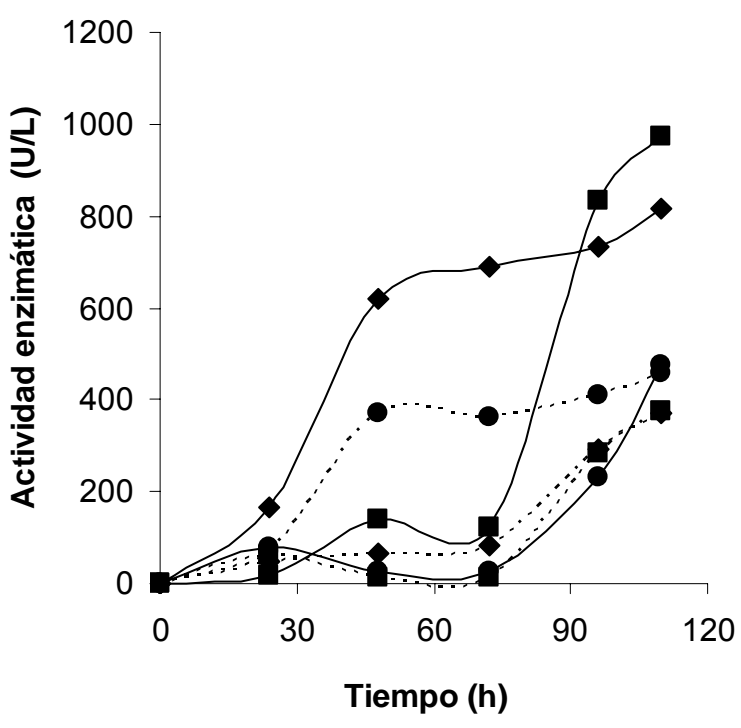

Fig. 3: Efecto de la adición de pulsos de glucosa sobre la producción de la actividad queratinasa por en CML (línea sólida) y CMS (línea punteada). Los niveles de glucosa fueron: $1(\diamond), 5$ (匹) y 10 $(\bullet) \mathrm{g} / \mathrm{L}$.

\section{CONCLUSIONES}

El presente trabajo de investigación ha sido desarrollado para evaluar el potencial del pelo de cerdo como fuente de inductores de la enzima queratinasa y precursores de compuestos aminados en dos sistemas de cultivo microbiano. El pelo de cerdo puede ser utilizado como una fuente de carbono y nitrógeno debido a las altas cantidades que contiene de ambos elementos. Finalmente, el microorganismo Rhizopus oyzae es capaz de crecer en el medio de fermentación utilizando como única fuente de carbono al pelo de cerdo, además de producir bajo un mecanismo de inducción la enzima queratinasa.

\section{AGRADECIMIENTOS}

Los autores agradecen el apoyo técnico del Dr. Juan Carlos Contreras Esquivel y del Dr. Raúl Rodríguez Herrera. Este proyecto fue parcialmente financiamiento por la U.A. de C.

\section{REFERENCIAS}

AOAC. Official Methods of Analysis (13th Ed.). Association of Official Analytical Chemists, Washington, DC. USA. (1980).

Bascaran, V., V. Hardisson y A. Brana; Regulation of extracellular protease production in Streptomyces clavuligerus. App. Microbiol. Biotechnol: 34(2), 208-213 (1990).

Bertsch, A. y N. Coello; A biotechnological process for treatment and recycling poultry feathers as a feed ingredient. Bior. Technol.: 96(15),1703-1708 (2005)

Brandelli, A. y A. Rifle; Production of an extracellular keratinase from Chryseobacterium sp. growing on raw feathers. Electron. J. Biotechnol.: 8(1), 35-42 (2005).

Chandrasekaran, S. y S. Dhar; Multiple protease from Strptomyces moderatus. Isolation and purification of five extracellular protease . Arch. Biochem. Biophys.: 257(2), 395-401 (1986).

Cruz-Hernandez, M.A. y otros cuatro autores; Isolation and evaluation of tannin-degrading strains from the mexican desert. Zeitschrift für Naturforschung C: 60(11-12), 844-848 (2005). 
Farag, A. M. y M.A. Hassan; Purification, characterization and inmobilization of a keratinase from Aspergillus oryzae. Enzyme Microb. Technol.: 34(2), 85-93 (2003).

Grazziotin, A. y otros cuatro autores; Production of feather protein hydrolysate by keratinolytic bacterium Vibrio sp. kr2. Biores. Technol: 98(15), 3172-3175 (2007).

Ignatova, Z., G. Spassov y P. Nedkov; Isolation and partial characteriation of extracellular keratinase from a wool degrading thermophilic actinomycete strain Thermoactinomyces candidus. Can, J. Microbiol.: 45(3), 217-222 (1999).

Joo, H. S. Y otros cinco autores; Optimization of the production of an extracellular alkaline portease from Bacillus horikoshii. Proc. Biochem.: 38(2), 155-159 (2002).

Kembhavi A., A. Kulkami y A. Pant; Salt Tolerant and thermostable alkaline protease from Bacillus subtillis NCIM No 64. Appl. Biochem. Biotechnol.: 38(1-2), 83- 92 (1993).

Mahan, D.C. y R.G. Shields; Essential and nonessential amino acid composition of pigs from birth to 145 kilograms of body weight, and comparison to other studies. J. Anim. Sci.: 76(1), 513-521 (1998).

Malathi, S. y R. Chakraborty; Production of alkaline protease by a new Aspergillus flavus isolate under solid-substrate fermentation conditions for use as a depilation agent. Appl. Environ. Microbiol.: 57(3), 712-716 (1991).

Muro, T. y otros cuatro autores; Purification and some properties of protease 1 having transfer actino from Streptomyces griseus var. alcalophilus. Agric. Biol. Chem.: 55(2), 307-314 (1991).

Mushin, T.M. y R.B. Hadi; Degradation of keratin substrates by fungi isolated from sewage sludge. Mycopathologia: 154(4), 185-189 (2001).

Ozawa, S., K. Sato. e Y. Endo; Repeated batch production of alkaline protease by solid-state fermentation using urethane foam as carriers. Bioproc. Biosyst. Eng.: 14(1), 63-68 (1996).

Pokorny, M. y otros cinco autores; Streptomyces rimosus extracellular proteases. 1. Characterization and evaluation of various crude preparations. Europ. J. Appl. Microbiol. Biotechnol.: 8(1-2), 81-90 (1979).

Renko, M., M. Pokorny, Lj. Vitale, y V. Turk; Streptomyces rimosus extracellular proteases. 2. Isolation and characterization of serine alkaline proteinase. Europ. J. Appl. Microbiol. Biotechnol.: 11(1), 166-171 (1981).

Renko, M., Lj. Vitale. M. Kokalj, y M. Pokorny; Streptomyces rimosus extracellurar proteases. 4 , Trypsin-like proteinase. Appl. Microbiol. Biotechnol.: 31(1), 38-44 (1989).

Santos , R. y otros cuatro autores; Keratinolytic Activity of Aspergillus Fumigatus Fresenius. Current Microbiol.: 33(6), 364-370 (1996).

Suntornsuk, W. y Suntornsuk, L.; Feather degradation by Bacillus sp FK 46 in submerged cultivation. Biores. Technol.: 86(3), 239-243 (2003).

Vitale, L. y otros cuatro autores; Streptomyces rimosus extracellular proteases. 3. Isolation and characterization of leucine aminopeptidase. Appl. Microbiol. Biotechnol: 23(6), 449-455 (1986).

Yeoman, K. H. y C. Edwards; Protease production by Streptomyces thermovulgaris grown on repemeal-derived media. J. Appl. Bacteriol.: 77(3), 264-270 (1994). 